\title{
A Low-Voltage Flexible Cascade $\Sigma \Delta$ Modulator for Beyond-3G Wireless Telecom
}

\author{
Alonso Morgado, Rocío del Río and José M. de la Rosa \\ Instituto de Microelectrónica de Sevilla - IMSE-CNM (CSIC/Universidad de Sevilla) \\ Edif. CICA-CNM, Avda. Reina Mercedes s/n, 41012- Sevilla, SPAIN \\ Phone: +34 95 5056666, Fax: +34 95 5056686, E-mail: \{alonso|rocio|jrosa\}@imse.cnm.es
}

\begin{abstract}
This paper presents a new adaptable cascade $\Sigma \Delta$ modulator architecture for low-voltage multi-standard applications. It uses two reconfiguration strategies: a programmable global resonation scheme and a variable loop-filter order. These techniques are properly combined in a novel topology that allows to increase the effective resolution in a given bandwidth, whereas keeping relaxed output swing requirements and high robustness to mismatch and to non-linearities of the amplifiers. Time-domain simulations including main circuit-level effects are shown to demonstrate the benefits of the presented modulator when it is configured to cope with the requirements of GSM, UMTS, WLAN and Wi-Max. ${ }^{\dagger 1}$
\end{abstract}

\section{INTRODUCTION}

Sigma-Delta Modulators $(\Sigma \Delta \mathrm{Ms})$ are very suited for the

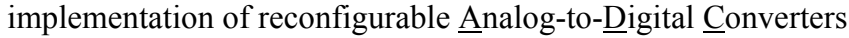
(ADCs) required for the fourth generation (4G or Beyond-3G) of wireless hand-held devices [1]. Based on the combined use of oversampling and noise shaping, $\Sigma \Delta \mathrm{Ms}$ adapt their dynamic ranges to different specifications with large hardware re-use, reduced power consumption and at the lowest cost [2]-[9].

Although changing the sampling frequency has been the reconfiguration strategy most commonly applied, the mandatory

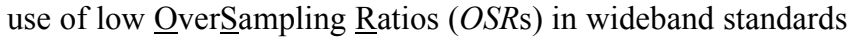
has forced $\Sigma \Delta \mathrm{Ms}$ to achieve the required dynamic ranges by resorting to different strategies, including: high-order shaping [2][6][8][9], switchable cascade topologies [5][9]; programmable notches within the signal band [2][8]; multi-bit embedded quantizers [3][7][9]. However, the efficient implementation of these techniques requires to explore new $\Sigma \Delta \mathrm{M}$ topologies capable of adapting their performance to as many specifications as possible while keeping high robustness to circuit errors.

The modulator presented in this paper is based on three strategies to adapt its functionality to different specifications. First, the modulator loop-filter order can be varied according to the required performance by connecting or disconnecting low-order stages in a cascade topology. Second, resonation is used to optimally distribute the zeroes of the Noise Transfer Function (NTF) within the required signal bandwidth. Third, unity Signal Transfer Function (STF) is employed in all stages of the cascade to reduce the amplifier output swing requirements [10]. The above-mentioned strategies are combined in a novel reconfigurable cascade $\Sigma \Delta \mathrm{M}$ topology that maximizes the effec-

$\dagger 1$. This work has been supported by the Spanish Ministry of Science and Education (with support from the European Regional Development Fund) under contract TEC2007-67247-C02-01/MIC, the Spanish Ministry of Industry, Tourism and commerce (FIT-330100-2006-134 SPIRIT) and the Andalusian Ministry of Innovation, Science and Enterprise under contract TIC-2532. tive resolution within a given bandwidth, with reduced circuit complexity, low sensitivity to element mismatches and high robustness with respect to opamp non-linearities.

\section{PROPOSED MODULATOR ARCHITECTURE}

Fig. 1 shows the proposed modulator architecture. It consists of a reconfigurable cascade topology that makes use of two concepts, namely: unity STF [10] and global resonation [11]. The former is implemented by the feedforward paths that connect the input of each stage with the corresponding quantizer inputs, whereas global resonation is achieved thanks to the inter-stage paths (highlighted in Fig.1) that feeds back a delayed scaled version of the last-stage quantization error at the input of the first-stage quantizer. One important advantage of the proposed modulator is that, on the contrary to the one reported in [11], only Forward-Euler (FE) integrators are required, thus making

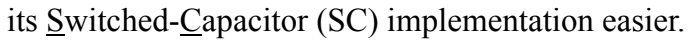

The modulator includes a number of switches to configure the NTF according to the required performance. On the one hand, by connecting the switchable error feedback paths from the last to the first stage - with switches $S_{r}$ and $S_{L}$ in ON mode - the global resonation effect is enabled. On the other hand, the order of the cascade is also reconfigurable. Both reconfiguration strategies, i.e. adaptive global resonation and NTF order, can be used independently, thus giving more flexibility to the $\Sigma \Delta \mathrm{M}$ to adapt its functionality to the required performance. In a practical application, those components which are not used can be turned off (by using a power-down control) in order to save power consumption.

In case resonation is enabled and considering a linear model for the embedded quantizers, it can be shown that the NTF of the proposed architecture is given by:

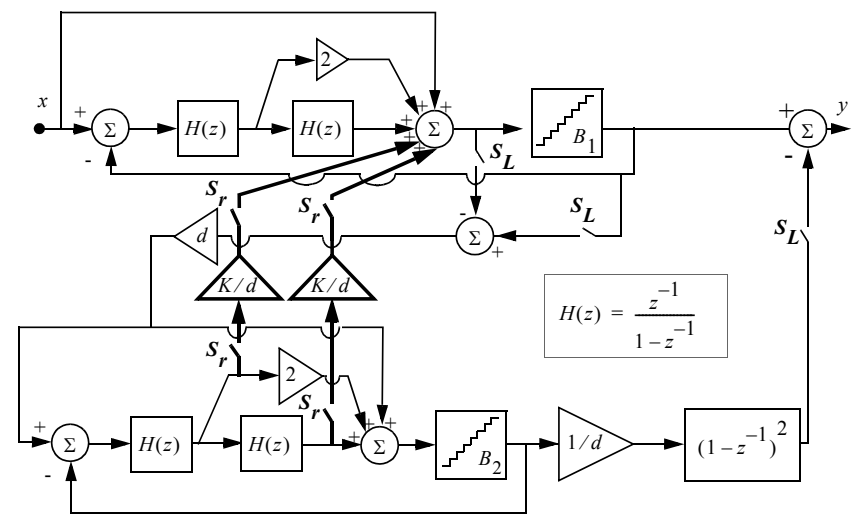

Figure 1. Proposed reconfigurable $\Sigma \Delta \mathrm{M}$ topology. 


$$
\operatorname{NTF}(z)=\frac{-\left(1-z^{-1}\right)^{2} \cdot\left[1-(2-K) \cdot z^{-1}+z^{-2}\right]}{d}
$$

where $d$ stands for the inter-stage gain. Note that two of the NTF zeroes are a function of $K$, whose value can be optimally chosen

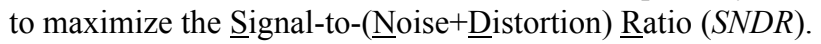

This effect is illustrated in Fig. 2 for an OSR of 4 and embedded quantizers with 4-bit resolution. The optimum values of $K / d$ for an inter-stage gain of 1 and 4 are roughly 0.4 and 0.1 , respectively implemented by capacitor ratios of $2 / 5$ and $1 / 10$. If there is no signal scaling along the cascade $(d=1)$, the resolution is increased in $11 \mathrm{~dB}$, whereas if $d=4$, an increment of $23 \mathrm{~dB}$ is achieved.

For the sake of completeness, Fig.3(a) shows the optimal location of the NTF zeroes in the unity-circle for oversampling ratios of 4,8 and 16. The effect on the noise shaping is depicted in Fig.3(b) by representing the NTF for the different cases together with their corresponding signal Band్idths ( $B W \mathrm{~s})$.

Note that an increase of $d$ (to reduce the quantization noise) yields a reduction of the feedback coefficient $K / d$. In practice, this results in a smaller capacitor ratio, which makes the electrical implementation more difficult and prone to circuit non-idealities. However, the value of $K / d$ can be easily implemented if the oversampling ratio is low enough because -as Fig.3(a) illustrates - a reduction of the $O S R$ means an increase of $K$. Therefore, the resonation provided by the proposed architecture is specially suited for wideband applications with low $O S R$.

\section{CASE STUDY: APPLICATION TO 4G WIRELESS TELECOM}

In order to show the reconfigurability of the proposed $\Sigma \Delta \mathrm{M}$ architecture, a case study is presented considering the specifications and standards listed in Table I. Global resonation is used only in WLAN mode. In this case, the rounded optimal feedback coefficient is $K / d=0.1$, resulting in a shift of two zeroes of the NTF from 0 to $0.8 \pm j \cdot 0.6$. Note that thanks to this optimum distribution of the NTF zeroes - together with the employment of an inter-stage scaling $d=4-$, the in-band noise has already been minimized, as shown in Fig. 2(b).

TABLE I

SPECIFICATIONS OF THE CASE STUDY

\begin{tabular}{|c|c|c|c|c|}
\hline Standard & GSM & UMTS $^{\mathbf{a}}$ & Wi-Max & WLAN \\
\hline \hline$S N D R(\mathrm{bit})$ & 14 & 12 & 8 & 10 \\
\hline$B W(\mathrm{MHz})$ & 0.2 & 4 & 20 & 20 \\
\hline
\end{tabular}

a. Although the bandwidth of UMTS is $3.84 \mathrm{MHz}$, it has been expanded to $4 \mathrm{MHz}$ in order to easily implement the sampling frequency.
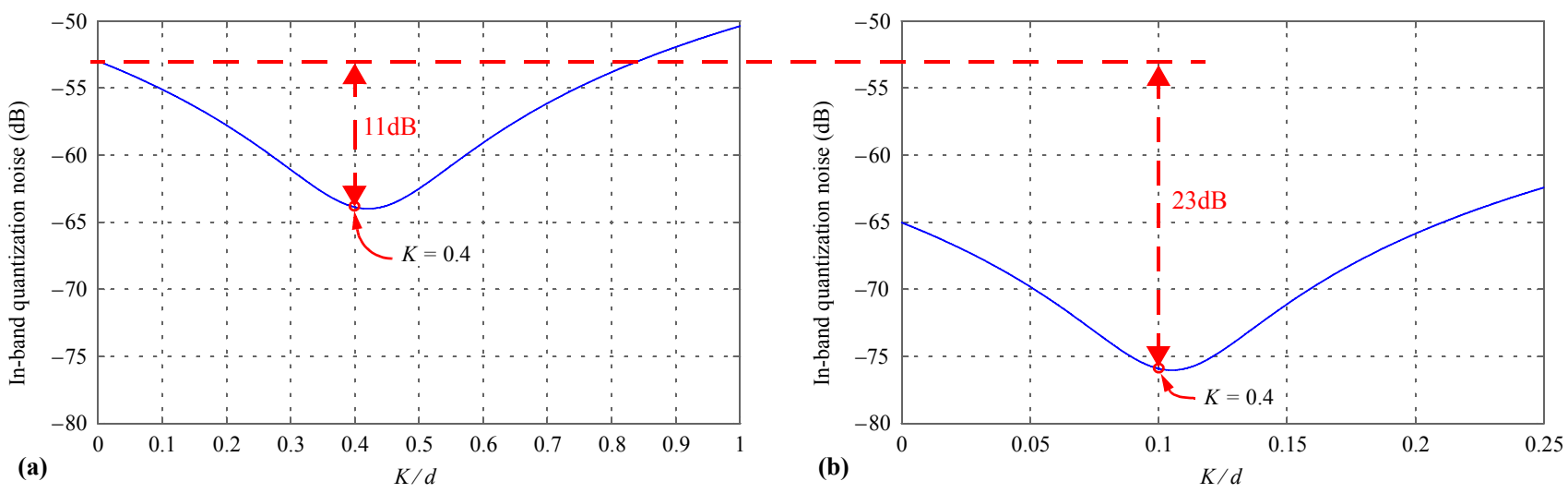

Figure 2. Variation of the in-band quantization noise with $K / d$ for: (a) $d=1$, (b) $d=4\left(O S R=4\right.$ and $\left.B_{1}=B_{2}=4\right)$.
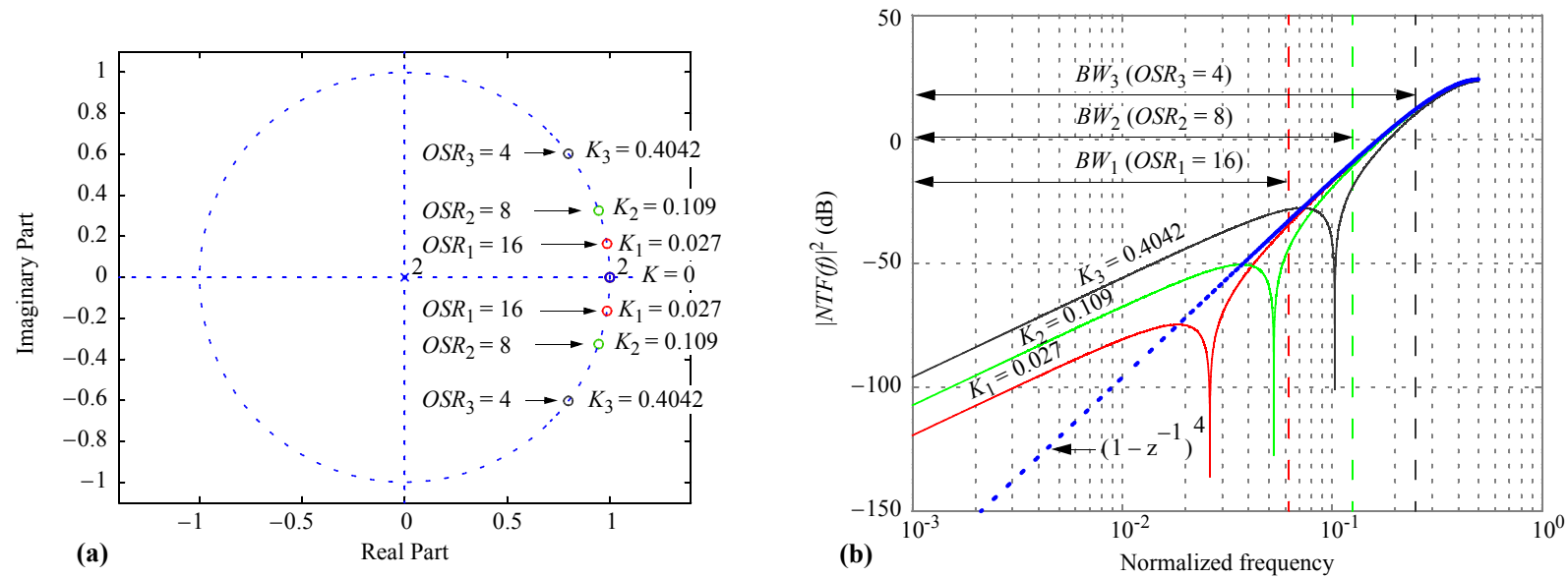

Figure 3. Optimum distribution of NTF zeroes in the proposed resonation-based $\Sigma \Delta \mathrm{M}$ for different oversampling ratios:

(a) Location of zeroes in the unit circle, (b) NTF vs. frequency $(d=1)$. 
Table II shows the values of the modulator design parameters that are reconfigured for the different operation modes, namely: NTF order $(L)$, resonation coefficient $(K / d)$ and $O S R$. Note that in those operation modes in which no resonation is used (GSM, UMTS and Wi-Max), the switches responsible for the last-stage error feedback ( $S_{r}$ in Fig. 1) are disconnected. Additionally, in GSM mode only the first stage is working —with switches $S_{r}$ and $S_{L}$ disconnected- whereas all the integrators and comparators of the second stage are turned off. Note that different oversampling ratios —and their corresponding sampling frequencies $\left(f_{s}\right)$ - are employed in order to cope with the required resolution of each signal bandwidth.

\section{SIMULATION RESULTS}

Several behavioral simulations have been performed using SIMSIDES, a Simulink-based time-domain simulator for $\Sigma \Delta$ modulators [12]. In all operation modes, 4-bit internal quantizers and a $1-\mathrm{V}$ reference voltage were considered. Additionally, $k T / C$ noise, sampled by 0.25 -pF sampling capacitors, is introduced at the first integrator in all simulations. The embedded Digital-to- $\underline{\text { Analog }}$ Converters (DACs) are assumed linear, although dynamic element matching will be required in practice at least in the first stage of the cascade.

Fig. 4 shows the modulator output spectra for all the operation modes. In GSM [Fig. 4(a)], the in-band error is dominated by thermal noise, whereas in UMTS [Fig. 4(b)] the larger error term is not clearly defined between thermal or quantization noise. The effect of resonation can be clearly distinguished by comparing Fig. 4(c) and (d), corresponding to Wi-Max and WLAN, respectively. Note that in WLAN, resonation reduces the in-band quantization error power as compared to Wi-Max. This is illustrated in Fig. 5 where the SNDR is represented versus the input signal amplitude. Note that the effective resolution for WLAN is approximately $9 \mathrm{~dB}$ larger than that for Wi-Max.

The sensitivity to noise leakages due to capacitor mismatch has been studied on the basis of Monte Carlo simulation. Fig. 6 shows the SNDR at -6dBFS obtained for a 50-run Monte Carlo simulation considering a standard deviation of $0.1 \%$ in all capacitors. The average $[m$ (bits)] and standard deviation $(\sigma)$ of the resulting effective modulator resolutions are also shown for each operation mode. Note that the use of resonation in the WLAN mode introduces no appreciable performance degradation when compared to the rest of operation modes.

TABLE II.

RESONATION COEFFICIENT, MODULATOR ORDER, OSR AND $d$

\begin{tabular}{|l|c|c|c|c|}
\hline Standard & GSM & UMTS & Wi-Max $^{\text {a }}$ & WLAN \\
\hline \hline Order $(L)$ & 2 & \multicolumn{3}{|c|}{4} \\
\hline Inter-stage gain $(d)$ & ---- & \multicolumn{3}{|c|}{4} \\
\hline$K$ & \multicolumn{3}{|c|}{0 (No resonation) } & 0.4 \\
\hline$K / d$ & \multicolumn{3}{|c|}{0 (No resonation) } \\
\hline$O S R$ & 100 & 10 & 4 \\
\hline$f_{S}(\mathrm{MHz})$ & 40 & 80 & \multicolumn{3}{c|}{160} \\
\hline
\end{tabular}

a. Note that resonation could also be employed in this case if a larger resolution is demanded. (a)

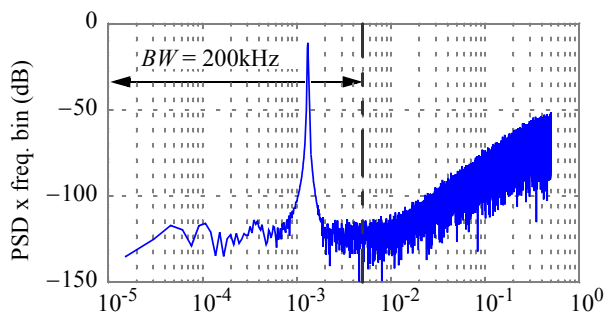

(b)

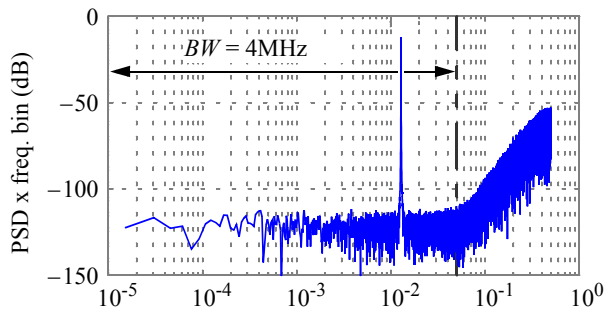

(c)

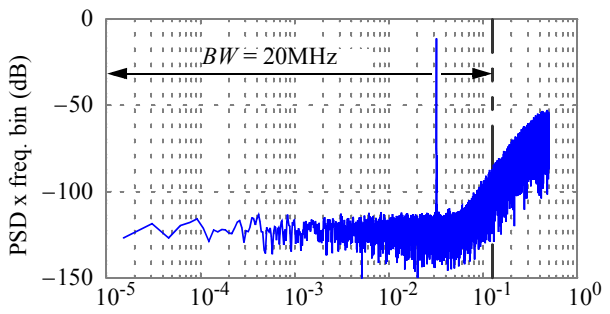

(d)

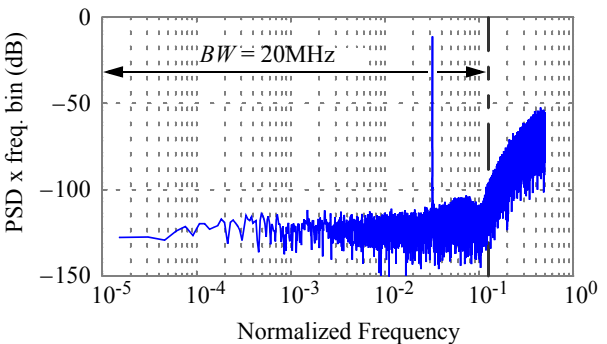

Figure 4. Modulator spectra of the different modes: (a) GSM, (b) UMTS, (c) Wi-Max, (d) WLAN (-6dBFS input level, 64k-point FFTs)

Fig. 7 shows the effect of the amplifiers finite DC gain on the modulator effective resolution for all the operation modes. Note that the impact of the amplifiers gain on the modulator performance is considerably lower in GSM when compared to the rest of standards. This is due to the topology used for the $\Sigma \Delta \mathrm{M}$ in the former case (single-loop), which does not present the sensitivity to noise leakages inherent to cascade topologies (used in

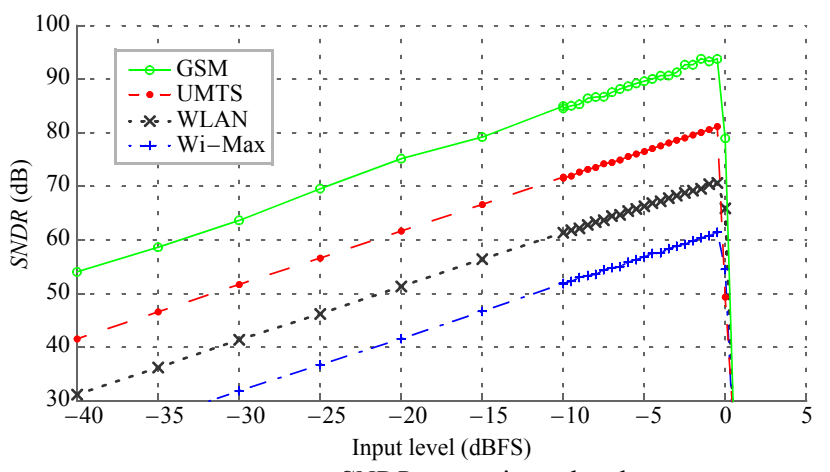

Figure 5. $S N D R$ versus input level. 
the remaining standards). In the case of UMTS and Wi-Max, there is no appreciable degradation for amplifiers gains above $40 \mathrm{~dB}$. This value raises to $50 \mathrm{~dB}$ for WLAN, which is still not a demanding requirement in practice.

An appealing advantage that comes from the use of unity STFs is the subsequent reduction of the amplifiers output swing. This is illustrated in Fig. 8 by plotting the histogram of the integrator outputs obtained from the operation of the proposed modulator in all operation modes. Note that the required output swing is only $\pm 0.15 \mathrm{~V}$ for the 1 st and 3rd operational amplifiers $\left(\mathrm{OA}_{1}\right.$ and $\left.\mathrm{OA}_{3}\right)$, whereas it reduces to $\pm 0.10 \mathrm{~V}$ for the $2 \mathrm{nd}$ and 4 th ones $\left(\mathrm{OA}_{2}\right.$ and $\left.\mathrm{OA}_{4}\right)$. This translates into an excellent linearity behavior for the proposed architecture.

The latter is illustrated in Fig.9 by plotting the effect of the opamp gain non-linearity on the modulator $S N D R$. In this simulation, a finite gain of $55 \mathrm{~dB}$ is considered for all amplifiers,

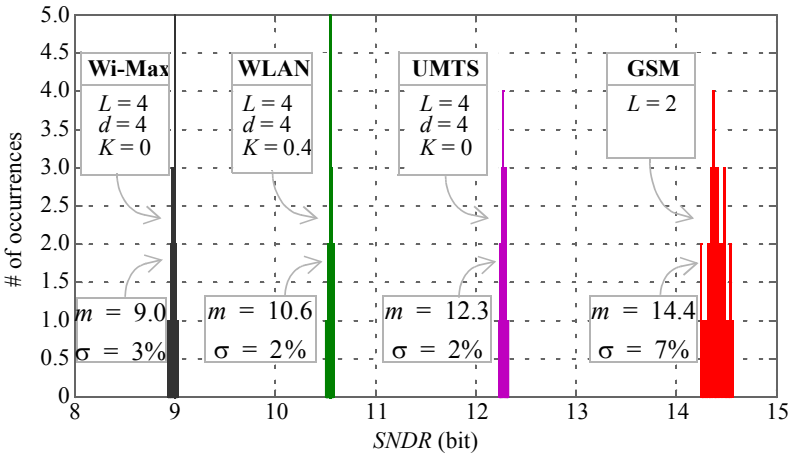

Figure 6. $S N D R$ variation considering a $0.1 \%$ capacitor mismatch (-6dBFS input level, 50-run Monte Carlo simulation).

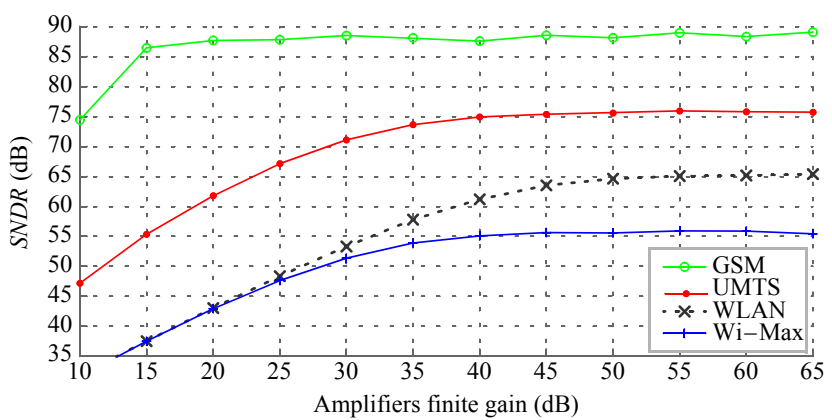

Figure 7. $S N D R$ vs. amplifiers finite gain (-6dBFS input level).

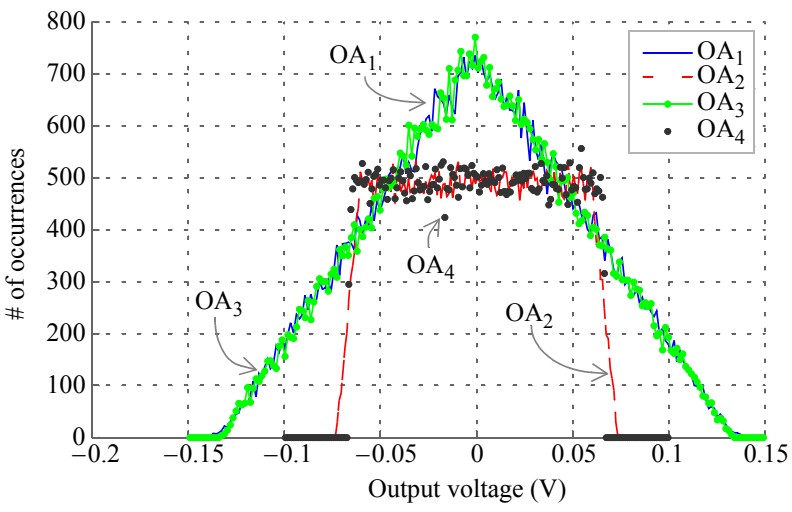

Figure 8. Output swing requirements.

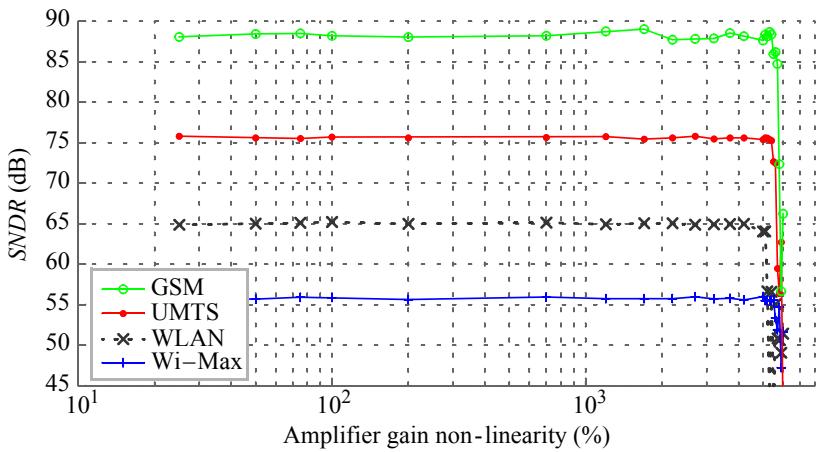

Figure 9. $S N D R$ vs. non-linearity of the amplifier gain (-6dBFS input level, 55-dB amplifier gain).

while varying the second-order non-linearity of the amplifier gain in the first stage amplifiers. Note that the robustness of the proposed $\Sigma \Delta \mathrm{M}$ to non-linearities is quite high, even if resonation is used to increase the effective modulator resolution.

\section{CONCLUSIONS}

A new multi-functional cascade $\Sigma \Delta \mathrm{M}$ architecture has been presented. This architecture is capable to reconfigure the order of the quantization noise filtering and to resonate through switchable feedback inter-stage paths. Additionally, it achieves high linearity with reduced output swing requirements by using unity STF. These characteristics make the proposed modulator very appropriate for the implementation of low-voltage multi-mode $\mathrm{A} / \mathrm{D}$ conversion, where the resolution of wideband standards can be easily increased by means of adaptive global resonation. A case study covering GSM, UMTS, Wi-Max and WLAN standards has been discussed in order to show the capabilities of the proposed modulator to perform the A/D conversion in future $4 \mathrm{G}$ wireless multi-standard hand-held devices.

\section{REFERENCES}

[1] X. Li and M. Ismail: Multi-standard CMOS wireless receivers: analysis and design. Kluwer Academic Publishers, 2002.

[2] T. Burger et al.: "A $13.5 \mathrm{~mW} 185-$ Msample/s $\Delta \Sigma$ Modulator for UMTS/GSM Dual-Standard IF Reception". IEEE J. of Solid-State Circuits, pp. 1868-1878, Dec. 2001.

[3] T.M.R. Miller et al.: "A Multibit Sigma-Delta ADC for Multimode Receivers". IEEE J. of Solid-State Circuits, pp. 475-482, March 2003.

[4] G. Gomez et al:: "A $1.5 \mathrm{~V} 2.4 / 2.9 \mathrm{~mW} 79 / 50 \mathrm{~dB}$ DR $\Sigma \Delta$ Modulator for GSM/WCDMA in 0.13 $\mu \mathrm{m}$ Digital Process". Proc. ISSCC, pp. 242-490, 2002.

[5] A. Dezzani et al:: "A 1.2-V Dual-Mode WCDMA/GPRS $\Sigma \Delta$ Modulator”. Proc. ISSCC, pp. 58-59, 2003.

[6] J.H. Shim et al.: "A Third-Order $\Sigma \Delta$ Modulator in 0-18-um CMOS With Calibrated Mixed-Mode Integrators". IEEE J. of Solid-State Circuits, pp. 918-925, April 2005.

[7] J. Lim et al.: "A Low-Power Sigma-Delta Modulator for Wireless Communication Receivers using Adaptive Biasing Circuitry and Cascaded comparator scheme". Analog Integrated Circuits and Signal Processing, pp. 359-365, Sept. 2006.

[8] T. Christen et al.: "A 0.13um CMOS EDGE/UMTS/WLAN Tri-Mode $\Sigma \Delta$ ADC with -92dB THD”. Proc. ISSCC, pp. 240-241, 2007.

[9] A. Morgado et al.: "Design of a 130-nm CMOS Reconfigurable Cascade $\Sigma \Delta$ Modulator for GSM/UMTS/Bluetooth”. Proc. ISCAS, vol. 1, pp. 725-728, May 2007.

[10]J. Silva et al.: "Wideband low-distortion delta-sigma ADC topology". IEE Electronics Letters, vol. 37, pp. 737-738, June 2001.

[11]M. Sanchez-Renedo et al.: "A 2-2 Discrete Time Cascaded $\Sigma \Delta$ Modulator With NTF Zero Using Interstage Feedback". Proc. ICECS, pp. 954-957, 2006.

[12]J. Ruíz-Amaya et al.: "High-level synthesis of switched-capacitor, Switched-Current and Continuous-Time $\Sigma \Delta$ Modulators Using SIMULINK-Based Time-Domain Behavioral Models". IEEE Trans. on Circuits and Systems-I, vol. 52, pp. 1795-1810, Sept. 2005. 